\title{
The Wuhan Pneumonia outbreak: High isoleucine and high valine plus glycine contents are features of the proteins of 2019- nCoV virus
}

Sirui Yan ${ }^{1}$, Yulin Wan ${ }^{1}$, Ying Zhang ${ }^{2}$, Shanshan $\mathrm{An}^{1}$, Kaiqiao Yang ${ }^{1}$, Huan $\mathrm{Xu}^{3}$, Qiuyun $\mathrm{Liu}^{1, *}$

${ }^{1}$ State Key Laboratory of Biocontrol, Biomedical Center, Guangdong Provincial Key Laboratory of Improved Variety Reproduction in Aquatic Economic Animals, School of Life Sciences, Sun Yat-sen University, Guangzhou 510275, China.

${ }^{2}$ Guangzhou Center for Disease Control and Prevention, Guangzhou 510440, China.

${ }^{3}$ School of Electronic Information and Automation, Guilin University of Aerospace Technology, Guilin 541004, Guangxi, China.

Sirui Yan and Yulin Wan made equal contributions to this work.

*Corresponding author: Qiuyun Liu, Ph.D, School of Life Sciences, Sun Yat-sen University, Guangzhou 510275, China. Tel: +86 (020)84110296; E-mail: 1sslqy@mail.sysu.edu.cn 


\begin{abstract}
The current pneumonia epidemic in China could evolve into a pandemic on a global scale if not effectively contained. The $2019-\mathrm{nCoV}$ possesses a 61 -amino acid open reading frame resembling SARS-CoV virulence factor - ORF6 peptide. The isoleucine content is $15.9 \%$ in ORF6 of SARS-CoV versus $16.4 \%$ of that in $2019-\mathrm{nCoV}$. Given the proton affinity in the carbonyl oxygen in isoleucine, augmented proton traffic can enhance proton-ion antiport and prompt cell swelling. As the content of essential amino acids in the open reading frame of $2019-\mathrm{nCoV}$ reaches $57.4 \%$, a starch/vitamin diet served for short period of time does not give rise to essential amino acids and halts virion production, which could be adopted as prophylactic approach of many viral infections. Plant-based diet or fasting/boiled rice water can also minimize the intake of essential amino acids or all amino acids respectively. Calorie restriction has been confirmed in animal studies to extend lifespan, and its underlying mechanism is not fully known. Furthermore, several proteins of 2019-nCoV possess high valine plus glycine content, which is implicated in heart disease.
\end{abstract}

\title{
Keywords
}

2019-nCoV; Proton affinity; Calcium oxalate; Isoleucine, Valine; Glycine; Prophylactic approach 


\section{Introduction}

The current outbreak in Wuhan, China, could develop into a pandemic on a global scale if not effectively controlled. The World Health Organization named the Wuhan BetaCoV as 2019-nCoV. Based on available data, the mortality rate is significantly higher in men than in women. The infection rate is lower in children and teenagers than in adults.

\section{Results and discussion}

The 2019-nCoV has a 61-amino acid open reading frame resembling SARS-CoV virulence factor - the 63-residue ORF6 protein. The basic amino acid content is slightly higher in ORF6 of SARS-CoV with $11.1 \%$ versus $9.8 \%$ in its counterpart of Wuhan strain nCoV-2019 (WIV02) (Fig. 1) (Zhou et al., 2020, Corman et al., 2020, Huang et al., 2020, Wang et al., 2020), whereas the isoleucine content is relatively close (15.9\% in ORF6 of SARS-CoV versus $16.4 \%$ of that in 2019-nCoV with identical number of isoleucine residues in the two open reading frames (orf)). However, the isoleucine residues are more packed in a shorter segment in SARS-CoV ORF6 protein, allowing more robust proton relay as isoleucine displays proton affinity (Gu et al., 1994, He et al., 2017). High fever and metabolic acidosis are two of the major symptoms in severe patients with 2019-nCoV infections, and are related to hydrogen bonding and proton traffic. The positively charged basic amino acids attract anions such as $\mathrm{Cl}^{-}$, enhancing the formation of strong acids. Augmented proton traffic promotes proton-ion antiport and cell swelling. The intake of boiled red meat without higher protein structures confers slight cancer risks, and the $\beta$-amyloid plaques and neurofibrillary tangles correlate to the onset of Alzheimer's disease, suggesting that protein primary structures are implicated in disease etiology. The lower rate of 2019-nCoV in children can be at least partly explained by the less robust Krebs cycle in young kids generating less protons, consequently yielding less proton stress. Women also have less strong energy metabolism than men. The human transmissibility and the long latency period of the Wuhan strains may drive the spread of the virus to other regions of world, underscoring the urgency for preventive measures. As the percentage of essential amino acids in the 
small orf of 2019-nCoV reaches $57.4 \%$, a starch/vitamin diet served to the potentially exposed human subpopulation for short period of time does not give rise to essential amino acids such as isoleucine and lysine and reduces the viral load, which could be adopted as prophylactic approach of 2019-nCoV (Gan et al., 2018; Wan et al., 2019; He et al., 2018; Wahl et al., 2018). Peptides consisting of essential amino acids are often stressful (Tang et al., 2017). Plant-based diet, fasting/boiled rice water or sugar water/vitamins can be also used to minimize the intake of essential amino acids or all amino acids. During fasting or consuming sugar water, occasional limited cointake of protein-containing nutrients may relieve some stress generated by abnormal ion traffic via ionic bonds or secondary chemical bonding with proteins, peptides or amino acids. Some of these are tried and tested ancient antiviral remedies which ensured human survival and can be explained by modern science. Appearance of essential amino acids may be the consequence of natural selection against viral and perhaps some other diseases as flu-stricken individuals tend to lose appetite, particularly appetite toward meat which is high in essential amino acids. Fasting is also used in some individuals in western society to extend lifespan, and it accounts for the prolonged lifespan of Americans during the Great Depression, known as calorie restriction. These food regimens should be tested in clinical trials, and individuals need to be instructed by a physician.

Valine and glycine content of the orfs of 2019-nCoV is shown in Table 2. Since valine and glycine harbor affinity to divalent cations such as calcium, and isoleucine possesses proton affinity (Qi et al., 2018; Wan et al., 2019; Xing et al., 2017; Wang et al., 2017), valine or glycine compete with isoleucine and alleviate fever and other symptoms. However, high content of valine or/and glycine are implicated in heart disease and other disorders by the formation of insoluble and rigid calcium oxalate which are stressful to the body (Qi et al., 2018; Wan et al., 2019), which accounts for the higher mortality rates in these patients concomitant with 2019-nCoV infections. Patients with renal stones, constipation, heart disease, Alzheimer's disease, Prion disease, etc. need to be cautious of $2019-\mathrm{nCoV}$ as insoluble calcium salts may be present in their bodies which can be compounded by viral infections. The higher 
mortality rates in Wuhan and Hubei Province than elsewhere can be attributed to the low temperature in winter without heating, driving robust energy metabolism to produce an excess of oxalate which leads to the formation of calcium oxalate (Zhou et al., 2017; Shi et al., 2017). The low food intake mentioned above could minimize the generation of oxalate via the reduced energy metabolism. Injection of physiological saline (divalent cation-free $\mathrm{NaCl}$ ) may help dissolve insoluble and rigid calcium oxalate via the action of $\mathrm{Cl}^{-}$. RNA interference can be adopted to decrease the production of oxalate. All these regimens or treatments should be subjected to clinical trials before antiviral application.

\section{Conflict of interest statement}

The authors declare no conflict of interests.

\section{Acknowledgements}

Q.L. was supported by grants from the Science and Technology Transformation Program of Sun Yat-sen University of China (33000-18843234), Guangzhou Science and Technology Program (201804010328) and Guangdong Science and Technology Program (2016B020204001). We are grateful to helpful discussions with Jiaming Zhang, Weiguo Cao and Yan Shi.

\section{Author Contributions}

QL contributed to the conception and design of the work. QL, SY, YW, YZ, SA, KY and HX contributed to the analysis and interpretation of data for the work. QL, SY and YW drafted the manuscript with input from all authors.

\section{References}

Corman V.M., Landt O., Kaiser M., et al., 2020. Detection of 2019 novel coronavirus (2019-nCoV) by real-time RT-PCR. Euro. Surveill. 25(3), pii=2000045.

Gan T., Fu M., Wu J., Wen L., Liu Q., 2018. How to design carbohydrate diet regimens 
for heart disease patients. Eur. J. Prev. Cardiol. 25(9), 979-980.

Gu Z., Zambrano R., McDermott A., 1994. Hydrogen bonding of carboxyl groups in solid-state amino acids and peptides: comparison of carbon chemical shielding, infrared frequencies, and structures. J. Am. Chem. Soc. 116, 6368-6372.

He J., Luo J., Xu Z., et al., 2017. High isoleucine content in the ORF6 peptide of SARS virus may contribute to virulence. Science. http://science.sciencemag.org/content/339/6125/1287/tab-e-letters

He R., He S., Li X., Shi Y., Liu Q., 2018. How can heart disease patients prevent complications from viral infections? Eur. J. Prev. Cardiol. 25(7), 758.

Huang C., Wang Y., Li X., et al., 2020. Clinical features of patients infected with 2019 novel coronavirus in Wuhan, China. Lancet. https://doi.org/10.1016/S0140$6736(20) 30183-5$

Qi J., Zhang X., Chen Z., Shi Y., Liu Q., 2018. Transgenic proteins rich in valine or glycine are concerns for heart disease patients. Eur. J. Prev. Cardiol. 25(8), 883884.

Shi, Y., Wan, Y., Zhou, Y., Ye, Z., Liu, Q. 2017. Why various wines reduce the risks of heart diseases. Eur. J. Prev. Cardiol., 24, 1646-1647. DOI: $10.1177 / 2047487317730171$.

Tang M., Weng S., Yu G., et al., 2017. RE: Sequence features of the virulence protein VP24 of Ebola virus. Science.

http://science.sciencemag.org/content/351/6279/1339/tab-e-letters

Wahl D., Solon-Biet S.M., Wang Q., et al., 2018. Comparing the effects of lowprotein and high-carbohydrate diets and caloric restriction on brain aging in mice. Cell Reports. 25(8), 2234-2243.e6.

Wan Y., Zhang J., Li X., Wang Y., Liu Q., 2019. Cellular states and secondary chemical bonding: a biochemical view of major human diseases. Biochem. Insights. 12, 1178626419877846 .

Wang C., Horby P.W., Hayden F.G., Gaoh G.F., 2020. A novel coronavirus outbreak of global health concern. Lancet. https://doi.org/10.1016/S0140-6736(20)30185-9

Wang Y., Hu X., Zhang J., Gao Y., Huang Y., Liu Q., 2017. Furthering our 
understandings on coding SNPs: $\beta$-branched amino acids in diseases. Science. http://science.sciencemag.org/content/350/6265/1262/tab-e-letters

Xing M., Zhang X., Wang Y., et al., 2017. RE: Chemical considerations on artificial protein designs. Science. http://science.sciencemag.org/content/352/6286/680/tabe-letters

Zhou P., Yang X., Wang X., et al., 2020. Discovery of a novel coronavirus associated with the recent pneumonia outbreak in humans and its potential bat origin. BioRxiv. https://doi.org/10.1101/2020.01.22.914952

Zhou, Y., Shi, Y., Wan, Y., Ye, Z., Liu, Q., 2017. Why the Mediterranean diet lowers the risk of heart disease. Eur. J. Prev. Cardiol. 24, 1788-1789. DOI: $10.1177 / 2047487317730653$.

Table 1 The amino acid content of ORF6 protein of SARS-CoV versus its counterpart of Wuhan strain nCoV-2019 (GenBank accession no.: MN908947.3).

\begin{tabular}{ccccc}
\hline & \multicolumn{2}{c}{ SARS_CoV } & \multicolumn{2}{c}{ Wuhan nCoV-2019 } \\
\cline { 2 - 5 } Amino Acid & $\begin{array}{c}\text { No. of } \\
\text { residues }\end{array}$ & Content $\%$ & $\begin{array}{c}\text { No. of } \\
\text { residues }\end{array}$ & Content \% \\
\hline Ala(A) & 2 & 3.2 & 1 & 1.6 \\
Arg(R) & 3 & 4.8 & 1 & 1.6 \\
Asn(N) & 2 & 3.2 & 4 & 6.6 \\
Asp(D) & 5 & 7.9 & 4 & 6.6 \\
Cys(C) & 0 & 0.0 & 0 & 0.0 \\
Gln(Q) & 2 & 3.2 & 3 & 4.9 \\
Glu(E) & 5 & 7.9 & 5 & 8.2 \\
Gly(G) & 0 & 0.0 & 0 & 0.0 \\
His(H) & 1 & 1.6 & 1 & 1.6 \\
Ile(I) & 10 & 15.9 & 10 & 16.4 \\
Leu(L) & 7 & 11.1 & 8 & 13.1 \\
Lys(K) & 3 & 4.8 & 4 & 6.6 \\
Met(M) & 3 & 4.8 & 3 & 4.9 \\
Phe(F) & 4 & 6.3 & 3 & 4.9 \\
Pro(P) & 3 & 4.8 & 1 & 1.6 \\
Ser(S) & 3 & 4.8 & 4 & 6.6 \\
Thr(T) & 3 & 4.8 & 3 & 4.9 \\
Trp(W) & 1 & 1.6 & 1 & 1.6 \\
Tyr(Y) & 2 & 3.2 & 2 & 3.3 \\
Val(V) & 4 & 6.3 & 3 & 4.9 \\
& & & &
\end{tabular}




$\begin{array}{ccccc}\text { Basic Amino } & 7 & 11.1 & 6 & 9.8 \\ \begin{array}{c}\text { Acids(R,K,H) } \\ \text { Acidic Amino }\end{array} & 10 & 15.9 & 9 & 14.8 \\ \text { Acids(D,E) } & & & & \\ \text { Essential Amino } & 35 & 55.6 & 35 & 57.4 \\ \text { Acids(K,W,F,M,T,I,L,V) } & 11 & 17.5 & 11 & 18.0 \\ \text { Leu/Phe(L,F) } & 63 & & 61 & \\ \text { Total } & 63 & \end{array}$


Table 2 Amino acid composition of Wuhan strain nCoV-2019 (GenBank accession no.: MN908947.3).

\begin{tabular}{ccccccc}
\hline & \multicolumn{5}{c}{ Partial amino acid composition \% } & \\
\cline { 2 - 6 } & & & & & Basic & Acidic \\
& $\mathrm{I}$ & $\mathrm{V}$ & $\mathrm{G}$ & $\mathrm{V}+\mathrm{G}$ & $\begin{array}{c}\text { amino } \\
\text { acids }\end{array}$ & amino acids \\
\hline ORF1ab & 5.2 & 8.5 & 6.0 & 14.5 & 11.42 & 11.01 \\
$\mathrm{~S}$ & 6.0 & 7.6 & 6.4 & 14.1 & 9.4 & 8.64 \\
ORF3a & 7.6 & 9.1 & 5.1 & 14.2 & 9.1 & 8.7 \\
$\mathrm{E}$ & 4.0 & 17.3 & 1.3 & 18.7 & 6.7 & 4.0 \\
M & 9.0 & 5.4 & 6.3 & 11.7 & 11.71 & 5.85 \\
ORF6 & 16.4 & 4.9 & 0.0 & 4.9 & 9.8 & 14.8 \\
ORF7a & 6.6 & 6.6 & 3.3 & 9.9 & 12.4 & 8.26 \\
ORF8 & 8.3 & 9.9 & 4.1 & 14.0 & 10.7 & 10.8 \\
$\mathrm{~N}$ & 3.3 & 1.9 & 10.3 & 12.2 & 15.3 & 8.6 \\
ORF10 & 7.9 & 10.5 & 2.6 & 13.1 & 5.3 & 2.6 \\
\hline
\end{tabular}

